\title{
Testicular Yolk Sac Tumor
}

National Cancer Institute

\section{Source}

National Cancer Institute. Testicular Yolk Sac Tumor. NCI Thesaurus. Code C8000.

A non-seminomatous malignant germ cell tumor arising from the testis. It affects

infants, young children, and postpubertal males. It is the most frequently seen testicular

neoplasm during childhood. The vast majority of patients present with an asymptomatic scrotal mass. The tumor mimics the yolk sac of the embryo and produces alpha-

fetoprotein (AFP). It metastasizes to distant anatomic sites. Prognostic factors relate to the clinical stage and the deg ree of AFP elevation. 